\title{
,Fieberhafte Steigerung'. \\ Zur Zuverlässigkeit des Erzählers in \\ Thomas Manns Doktor Faustus (1947)
}

\author{
Tom Kindt
}

„Erinnerungen sind nicht unzuverlässiger

als alles andere, was wir uns ausmalen.“

(Robert Menasse: Die Hauptstadt)

Zusammenfassung Der Beitrag rekonstruiert und problematisiert grundlegende Spielarten der verbreiteten These, dass Serenus Zeitblom, der fiktive Narrator in Thomas Manns „Doktor Faustus“, ein unzuverlässiger Erzähler ist. Die Abwägungen gelangen im Wesentlichen zu den folgenden drei Einschätzungen: 1) Aus der Ironisierung Zeitbloms im Werkzusammenhang lässt sich nicht auf seine Unzuverlässigkeit schließen. 2) Trotz wachsender Fabulierlust während des Erzählens legt er einen mimetisch zuverlässigen Bericht vor. 3) Trotz eines nach und nach geschärften Urteilsvermögens präsentiert er eine - in besonderer Weise axiologisch unzuverlässige Erzählung.

In Untersuchungen zum deutschsprachigen Roman nach 1945 findet Thomas Manns Doktor Faustus zumeist wenig Beachtung. Die Literaturgeschichtsschreibung folgt hier bis heute einer Sichtweise, die sich nach dem Erscheinen des Romans im Jahr 1947 rasch durchgesetzt hat. ${ }^{1}$ Schon vielen zeitgenössischen Lesern erschien das Werk nicht eigentlich zeitgenössisch, nicht als ein Dokument der eigenen Gegenwart, sondern als eine Art Schlussakte der historischen Periode, die mit dem Krieg zu Ende gegangen war. In diesem Sinne nannte etwa Erich von

${ }^{1}$ Der Roman erschien 1947, zunächst bei Bermann-Fischer in Stockholm und etwas später bei Suhrkamp in Frankfurt am Main.

T. Kindt $(\bowtie)$

Departement für Germanistik,

Universität Fribourg,

Freiburg, Schweiz

E-Mail: tom.kindt@unifr.ch 
Kahler den Roman 1948 eines der „terminal books“ (von Kahler 1948, S. 20)² des 20. Jahrhunderts, eine abschließende literarische Bestandsaufnahme zur Situation des modernen Menschen. ${ }^{3}$ Und Mann selbst hat einem entsprechenden Verständnis einigen Vorschub geleistet, indem er in seinem Werkstattbericht Die Entstehung des Doktor Faustus von 1949 erklärte, das Werk sei nicht zuletzt als „Kultur- und Epochen-Roman“ (Mann 1949, S. 438) über das Zeitalter zu verstehen, das zu Nationalsozialismus und Weltkrieg geführt habe.

Auch wenn es im Sinne solcher Einordnungen einleuchtet, Doktor Faustus im Kontext der Zwischenkriegs- und Exilzeit zu betrachten, so sollte dies doch nicht dazu verleiten, die Zusammenhänge zu unterschätzen, die zwischen dem Text und der Romanliteratur der Nachkriegszeit bestehen. Manns Werk über Das Leben des deutschen Tonsetzers Adrian Leverkühn, erzählt von einem Freunde ist fast zur Hälfte nach dem Zweiten Weltkrieg entstanden ${ }^{4}$ und sollte einigen Einfluss auf den deutschsprachigen Roman der 50er, 60er und 70er Jahre gewinnen. ${ }^{5}$ Alfred Andersch scheint dies früh vorausgesehen zu haben, in seiner Bestandsaufnahme Deutsche Literatur in der Entscheidung wies er Manns Werk schon drei Jahre nach Kriegsende eine eigentümliche Zwischenstellung zwischen Vor- und Nachkriegsmoderne zu: „Wir stehen mitten im Vorgang einer neuen Wandlung, und es macht die Größe Thomas Manns aus, daß er uns die Türe in einen Raum nicht versperrt, sondern öffnet, in den er selbst nicht mehr einzutreten vermag" (Andersch 1948, S. 18).

Das Verhältnis zwischen Manns Roman und der deutschsprachigen Nachkriegsliteratur - und mithin die Angemessenheit der Tür-Metapher von Andersch wird im vorliegenden Kontext nicht zu klären sein. Durch Betrachtungen zur Erzählanlage des Doktor Faustus soll im Folgenden aber zumindest versucht werden, eine nähere Bestimmung seiner Beziehungen zur Erzählpoetik des Nachkriegsromans vorzubereiten. ${ }^{6}$ Meine Einlassungen legen es nicht darauf an, eine ausgefeilte Interpretation von Manns Text $\mathrm{zu}$ entwickeln; sie werden sich auf die genaue Analyse eines einzigen, für das Verständnis des Romans freilich fundamentalen Aspekts seines erzählerischen Profils konzentrieren - auf die Untersuchung der narrativen Zuverlässigkeit des fiktiven Erzählers Serenus Zeitblom.

\footnotetext{
${ }^{2}$ Hervorhebung im Original.

${ }^{3}$ An anderer Stelle seines Essays bezeichnet von Kahler Manns Gesamtwerk als „terminal oeuvre“ und den Faustus-Roman als dessen „,final chapter“ (von Kahler 1948, S. 30).

${ }^{4}$ Manns Überlegungen zu einem Faustus-Projekt reichen bis 1904 zurück, ausgearbeitet und niedergeschrieben wird der Roman aber erst zwischen 1943 und 1947, vgl. zur Entstehungsgeschichte die Darstellung in Wimmer und Stachorski (2007, S. 11-59), und den Überblick in Vaget (2012, S. 443-445).

${ }^{5}$ Dies wird gelegentlich konstatiert (so etwa Mayer 1988, S. 65), scheint bislang aber nur sehr punktuell genauer untersucht worden zu sein (vgl. z. B. Neumann 2016, S. 154-159).

${ }^{6} \mathrm{Zu}$ den Verbindungen des Romans zur Moderne der Zwischenkriegszeit liegen bereits grundlegende Untersuchungen vor, vgl. z. B. Kaiser (2001) und Schmidt-Schütz (2003).
} 


\section{Manns Medium}

Dass Thomas Mann in seinem Werk immer wieder auf erzählerische Verfahren zurückgreift, die sich der Tradition unzuverlässiger Narration zurechnen lassen, ist in den zurückliegenden Jahren zunehmend in den Fokus der Forschung gerückt. Mittlerweile wird die Bedeutung erzählerischer Unzuverlässigkeit für alle Phasen und viele wichtige Texte des Mann'schen Euvres behauptet, von seinen Erzählungen der Zeit um 1900 über Werke wie Tod in Venedig oder Mario und der Zauberer bis hin zu seinen Romanen der Exil-, Kriegs- und Nachkriegsjahre wie Joseph und seine Brüder oder Felix Krull. ${ }^{7}$

Nicht für all diese Texte mag es überzeugend erscheinen, ihre je spezifische Erzählweise als Fall narrativer Unzuverlässigkeit einzustufen ${ }^{8}$ - für den Doktor Faustus liegt es aber sehr nahe. Erzähler des Romans ist der Altphilologe und ehemalige Gymnasiallehrer Serenus Zeitblom, der in Deutschland während des Zweiten Weltkriegs den Versuch unternimmt, das Leben des Komponisten Adrian Leverkühn, seines Jugendfreundes, von der Schul- und Studienzeit über eine genialische Schaffensphase bis zum syphilisbedingten Absinken in einen Zustand geistiger Umnachtung im Jahr 1930 darzustellen. ${ }^{9}$ Mit Zeitblom lässt Mann offenbar genau den Typ von Erzähler auftreten, der Wayne C. Booth eineinhalb Jahrzehnte später zur Einführung des Begriffs des ,unreliable narrator' veranlassen sollte, nämlich einen figürlich ausgestalteten Erzähler, dessen Werthaltungen sich von denen abzuheben scheinen, die durch das Werk im Ganzen ausgedrückt werden. ${ }^{10}$ Booths Definition in der Rhetoric of Fiction von 1961 lautete entsprechend: „I have called a narrator reliable when he speaks for or acts in accordance with the norms of the work [...], unreliable when he does not" (Booth 1961, S. 158 f.). ${ }^{11}$

Im Sinne dieser Bestimmung lässt sich schon Manns eigener Kommentar zur Erzählerfigur seines Romans als Hinweis auf deren ,unreliability“ verstehen, auch wenn ihm dieser oder ein vergleichbarer Begriff natürlich nicht zur Verfügung stand. In seiner Entstehungsgeschichte des Doktor Faustus beschreibt Mann seinen Erzähler Zeitblom als ein „Medium“, das er zwischen sich und die dargestellten Geschehnisse habe „schalten“ müssen, „um eine gewisse Durchheiterung des düsteren Stoffes zu erzielen und [...] seine Schrecknisse erträglich zu machen“ (Mann 1949, S. 430). Die Erzählanlage des Textes, so Mann weiter, beruhe auf der „komischen Idee“, „das Dämonische durch ein

\footnotetext{
${ }^{7}$ Für einen Überblick über die entsprechenden Debatten mit Hinweisen auf weitere Literatur vgl. Kindt (2015, S. 253).

${ }^{8}$ Vgl. hierzu etwa Löwe (2018).

${ }^{9}$ Wenn hier und im Folgenden von „Erzählern“ die Rede ist, sind stets „,fiktive Erzähler“ gemeint.

${ }^{10}$ Zum Kontext der Einführung des Konzepts vgl. Kindt (2008, S. 29-34).

${ }^{11}$ Hervorhebungen im Original.
} 
exemplarisch undämonisches Mittel“ (Mann 1949, S. 430) zu präsentieren, in anderen Worten: einen fiktiven Erzähler berichten zu lassen, der die Ereignisse in seinen Schilderungen zumindest tendenziell anders sieht, als es durch den Roman im Ganzen nahe gelegt wird.

Es kann angesichts dieser kompositorischen Idee nicht erstaunen, dass die erste explizite Einordnung von Zeitblom als ,unreliable narrator' bereits im Zusammenhang der Begriffsprägung erfolgt. Schon in der Rhetoric of Fiction führt Booth Doktor Faustus als Beispiel für ein unzuverlässiges Erzählen an, wie es im modernen Roman sehr verbreitet sei:

There have been $[\ldots]$ many self-conscious narrators in modern fiction, but they have almost all been dramatized as unreliable characters quite distinct from their authors. The narrators of Mann's Dr. Faustus [...], of Gides Les faux monnayeurs, and of Huxley's Point Counter Point, all engage in strongly implied praise for the works they are writing, but despite their pretensions, they are writing works rather strikingly different from the actual novels of Mann, Gide and Huxley. (Booth 1961, S. 205, Fn. 28) 12

Booths Einordnung des Faustus-Romans ist mittlerweile zur Standardsichtweise geworden. Mit dem Begriff des ,unzuverlässigen Erzählers' hat sich seit den 1970er Jahren ebenso in der Mann-Philologie ${ }^{13}$ wie allgemein in der Erzähltheorie $^{14}$ die Auffassung durchgesetzt, dass Serenus Zeitblom als ein solcher einzustufen ist.

Wie ein näherer Blick in die Faustus-Analysen zeigt, verbirgt sich hinter dieser weithin geteilten These eine Reihe unterschiedlicher Charakterisierungen des Erzählers Zeitblom, der zudem verschieden weite Konzepte von erzählerischer Unzuverlässigkeit zugrunde liegen. Wenn in den folgenden Abschnitten abgewogen wird, was von der Standardsichtweise $\mathrm{zu}$ halten ist, orientiert sich die Diskussion darum nicht an einzelnen Forschungsbeiträgen zum Doktor Faustus, sondern an wesentlichen Typen des Urteils, dass es sich bei Zeitblom um einen unzuverlässigen Erzähler handelt. Neben den Argumentationen für die betrachteten Urteilstypen werden dabei auch die jeweils vorausgesetzten Unzuverlässigkeitskonzepte auf dem Prüfstand stehen.

\section{Zeitbloms (Un-)Zuverlässigkeit}

Die weitgehende Einigkeit über die Unzuverlässigkeit des Faustus-Erzählers scheint wesentlich darin zu gründen, dass es sich bei ihm um einen nicht nur figural ausgestalteten, sondern zugleich ironisierten Narrator handelt. Zeitblom ist als ein ,Medium‘ angelegt, das die Ereignisse in ausgesprochen manierierter Form

\footnotetext{
${ }^{12}$ Hervorhebungen im Original.

${ }^{13}$ Vgl. etwa Viswanathan (1974), Riggan (1981), Kaiser (2001), Petersen (2007), Baier (2011) oder Ewen (2014).

${ }^{14}$ Vgl. z. B. Martinez und Scheffel (1999, S. 101), oder Fludernik (2009, S. 27).
} 
vermittelt, als ein Biograph, der sich über die Art seiner Schilderungen, ohne dies zu bemerken, selbst vorführt. Schon durch den altertümelnd-gestelzten Stil, in dem Mann seinen Erzähler berichten lässt, dadurch etwa, dass Zeitblom Begriffe wie „Morgentrank“ (Mann 1947, S. 251) oder „Schreibebrief“ (Mann 1947, S. 644) verwendet, statt ,Einstellung “,Bestallung“ (Mann 1947, S. 20) oder statt ,Hochzeitsnacht“ „Opferfest der Magdschaft“ (Mann 1947, S. 272) schreibt, wird die Aufmerksamkeit immer wieder vom Erzählten auf den Erzähler gelenkt und die Frage aufgeworfen, was von seinen Schilderungen zu halten ist. ${ }^{15}$

Anders als dies vielfach angenommen wird, folgt aus der ironischen Infragestellung eines Erzählers allerdings nicht notwendig, dass er im narratologischen Sinne als unzuverlässig einzustufen ist. Während sich jeder unzuverlässige Erzähler als ironisierter auffassen lässt, gilt das Umgekehrte nicht: Ein ironisierter Erzähler ist nur dann zugleich ein unzuverlässiger, wenn die Ironisierung bestimmte Merkmale seiner Ausführungen betrifft und anzeigt, dass seine Ereignisdarstellungen nicht adäquat sind oder seine Werthaltungen nicht repräsentativ für das Werk, dessen Teil er ist. Im ersten Fall ist der ironisierte Erzähler ,mimetisch unzuverlässig', im zweiten ,axiologisch unzuverlässig‘. Mit Blick auf Doktor Faustus bedeutet dies: Mit der Ironisierung Zeitbloms ist die Frage nach seiner Zuverlässigkeit nicht beantwortet - denn sie lässt die Möglichkeit offen, dass es sich bei ihm um einen Erzähler handelt, der einen zwar eigenwillig gestalteten, aber sachlich angemessenen und wertbezogen musterhaften Bericht liefert. ${ }^{16} \mathrm{Ob}$ dies so ist, soll nun gesondert betrachtet werden, zunächst wird die mimetische, dann die axiologische Zuverlässigkeit Zeitbloms diskutiert. ${ }^{17}$

Mimetische Zuverlässigkeit? Jüngere narratologisch ausgerichtete Beiträge zum Doktor Faustus gelangen recht einmütig zu dem Urteil, dass Zeitblom in mimetischer Hinsicht unzuverlässig erzählt. ${ }^{18}$ Zur Stützung dieses Urteils wird vor allem angeführt, dass er aus der selbstgewählten Rolle des Biographen falle. Immer wieder komme es in seiner Erzählung zu erheblichen „Kompetenzüberschreitungen" (Baier 2008, S. 113), denn er schildere nicht allein Ereignisse, von denen er durch eigene Anschauung oder das Zeugnis anderer Kenntnis habe, sondern auch solche, für die dies nicht gelte. In seiner genauen Analyse des Romans spricht Gerhard Kaiser deshalb von der „Lizenz zum (allwissenden)

\footnotetext{
${ }^{15} \mathrm{Zu}$ Zeitbloms Stil vgl. etwa auch Petersen (2007, S. 47-49).

${ }^{16}$ Vgl. Kindt (2008, S. 52): „Die Unterscheidung zwischen zuverlässigen und unzuverlässigen Erzählerfiguren fällt nicht mit der zwischen subjektiv berichtenden und objektiv berichtenden Sprechern zusammen“.

${ }^{17}$ Bezugspunkte sind dabei die Explikationsvorschläge für die Grundformen erzählerischer Unzuverlässigkeit in Kindt (2008), Köppe und Kindt (2011, 2014). - Nota bene: Voraussetzung der folgenden Abwägungen ist keine der in diesen Titeln oder andernorts vorgeschlagene konkrete Bestimmung ,unzuverlässigen Erzählens', sondern das prinzipelle Verständnis des Begriffs als literarhistorische Kategorie, als ein Konzept also, das sich nur unter Bezugnahme auf formale und funktionale Eigenschaften von Texten angemessen charakterisieren lässt.
}

${ }^{18}$ Vgl. insbesondere Kaiser (2001), Petersen (2007, 2008), Baier (2008, 2011). 
Erzähler, die sich Zeitblom im Verlauf des Romans selbst erteilt (bzw. die ihm sein Autor im wachsenden Maße zugesteht) und die an der Glaubwürdigkeit des Erzählten zunehmende Zweifel erregt“" (Kaiser 2001, S. 99). Ergänzt werden entsprechende Befunde häufig durch den Hinweis, dass Zeitblom überdies, entgegen seinen Ankündigungen, nicht nur Leverkühns, sondern auch die eigene Lebensgeschichte zu schildern versuche. Der Faustus-Erzähler führe die Leser, so fasst Christian Baier in diesem Sinne zusammen, im Hinblick auf Gattung und Gegenstand seiner Ausführungen in die Irre: „Serenus Zeitblom ist ein Romanerzähler, der sich als Biograph ausgibt, und das Werk, das er schreibt, ist im Grunde keine Biographie, oder weit mehr als das: Es ist zugleich und darüber hinaus seine eigene Autobiographie“ (Baier 2008, S. 125).

Die Beobachtungen, auf denen solche Einschätzungen beruhen, treffen zweifellos zu. Tatsächlich kommt Zeitblom in seiner Erzählung immer wieder auf seinen eigenen Lebensweg zu sprechen. Und tatsächlich ändert er im Verlauf der Ausführungen sein Erzählverhalten, um zunehmend in romanhafter Form auch von Ereignissen zu berichten, die er nach strengen historiographischen Maßstäben nicht auf diese Weise schildern dürfte. Was aber folgt hieraus für die Einordnung Zeitbloms? Nicht ohne Weiteres, dass er als mimetisch unzuverlässiger Erzähler zu charakterisieren ist. Es kommt mit Blick auf die Zuverlässigkeitsfrage entscheidend darauf an, wie man die beiden skizzierten Eigenschaften von Zeitbloms Erzählung fiktionsintern und fiktionsextern erklärt, ob man sie in der Fiktion überhaupt als ,Kompetenzüberschreitungen " deutet und, sofern man dies tut, welche Funktion man ihnen im Werkkontext zuschreibt. ${ }^{19}$

Eine entsprechende Erklärung der Erzählweise Zeitbloms hat zunächst in Rechnung zu stellen, dass er es erkennbar auf einen ganz bestimmten Typ von Lebensdarstellung anlegt; es geht ihm nicht um eine ,wissenschaftliche", sondern um eine ,literarische“ Biographie. ${ }^{20}$ Er wolle, so schickt Zeitblom voraus, die Vita Leverkühns aus der Perspektive des Jugendfreundes, guten Bekannten und treuen Bewunderers darstellen, werde sich aber zugleich bemühen, „den Standpunkt des komponierenden Künstlers einzunehmen und ihn mit der spielenden Besonnenheit eines solchen zu bewirtschaften“ (Mann 1947, S. 14). Im Sinne dieser - immer wieder in Erinnerung gerufenen - Ausrichtung der biographischen Erzählung scheint es grundsätzlich unproblematisch, dass Zeitblom auch von sich selbst oder von Ereignissen berichtet, von denen er nicht direkt, also durch eigene Beobachtung Kenntnis hat.

Dass Zeitblom mit literarischem Anspruch schreibt, kann Grundzüge seiner Ausführungen verständlich machen, es erklärt deren konkrete Ausgestaltung, insbesondere in späteren Abschnitten, aber nur unzureichend. Wie erwähnt, erzählt

\footnotetext{
${ }^{19}$ Vgl. zur Erklärung von erzählerischen Eigenwilligkeiten überblicksartig Kindt (2008, S. 60-62), und zur Motivierung der Eigenschaften von Texten und Filmen ausführlich Koch (2015), Kap. 5.

${ }^{20} \mathrm{Vgl}$. zu dieser Unterscheidung etwa Scheuer (1997).
} 
er hier in einer Weise, die selbst Im Rahmen einer , literarischen' Biographie bisweilen ungewöhnlich erscheint: Den ,Standpunkt des Künstlers` einzunehmen, heißt für Zeitblom in den betreffenden Passagen nicht mehr nur, in einem literarischen Stil zu schreiben; es schließt für ihn zunehmend die Möglichkeit ein, so zu erzählen, als unterläge sein Bericht keinerlei perspektivischen Restriktionen. Was hat es mit dieser Änderung der Erzählweise auf sich? Würde sie sich stillschweigend vollziehen, wäre es ohne Frage naheliegend, sie als eine Art Erschleichung von Allwissendheit und mithin als Hinweis auf Unzuverlässigkeit einzustufen. ${ }^{21}$ Im Doktor Faustus liegen die Dinge allerdings anders: Zeitblom kommentiert sein Erzählen fortlaufend und skizziert dabei eine Erklärung für dessen Eigentümlichkeiten, die bei der Beurteilung seiner mimetischen Zuverlässigkeit $\mathrm{zu}$ berücksichtigen ist.

Wie in seiner Erzählweise und seinen metanarrativen Kommentaren deutlich wird, gewinnt Zeitblom im Zuge seiner Schilderungen die Überzeugung, dass er für sich, da er retrospektiv berichtet und mit seinem Gegenstand intim vertraut ist, einen gleichsam unmittelbaren Zugang zu den dargestellten Ereignissen beanspruchen kann:

Dies ist kein Roman, bei dessen Komposition der Autor die Herzen seiner Personagen dem Leser indirekt durch szenische Darstellung erschließt. Als biographischer Erzähler steht es mir durchaus zu, die Dinge unmittelbar beim Namen zu nennen und einfach seelische Tatsachen zu konstatieren, welche auf die von mir darzustellende Lebenshandlung von Einfluß gewesen sind. (Mann 1947, S. 430)

Hinter dieser Auffassung Zeitbloms steht nicht die - von ihm ausdrücklich zurückgewiesene - Idee, er verfüge über ,,allwissende Autoreneinsichten“ (Mann 1947, S. 481). Sein Selbstverständnis als ,biographischer Erzähler" scheint vielmehr darauf zu beruhen, dass er Erlebnis und reflektierte Vorstellung als nahezu gleichwertige Wege betrachtet, bei Geschehnissen ,dabei zu sein'.22 Das ,Vorstellen“ oder ,Ausmalen' von Ereignissen, wie er selbst es nennt, gilt ihm als Spielart ihres Erlebens, zumindest dann, wenn es grundsätzlich auf eigener Beobachtung und guter Vertrautheit mit den vorgestellten Zusammenhängen beruht. ${ }^{23}$ In diesem Sinne behauptet er Augen- und Ohrenzeuge des Erzählten auch in den Fällen zu sein, in denen er es nicht, in der Realität', sondern , in der Phantasie ‘ miterlebt hat:

\footnotetext{
${ }^{21} \mathrm{Zu}$ einem entsprechenden nicht markierten Rollenwechsel eines fiktiven Erzählers vgl. z. B. Kindt (2007).

${ }^{22}$ Erstmals deutet sich dies in Zeitbloms Bericht über den Unterricht des jugendlichen Leverkühn an: „Ich bin ja bei diesen Unterrichtsstunden niemals zugegen gewesen und weiß davon nur vom Hörensagen, kann mir aber leicht das Verhalten meines Adrian dabei vorstellen" (Mann 1947, S. 55; Hervorhebung von mir, T. K.).

${ }^{23} \mathrm{Vgl}$. dazu: „Was soll ich weiter sagen? Ich bin zu alt und zu traurig, um eine Szene auszumalen, an deren Einzelheiten auch niemandem gelegen sein kann“ (Mann 1947, S. 646; Hervorhebung von mir, T. K.).
} 
Was $[\ldots]$ sich abspielte, und wie es sich abspielte, - ich weiß es, und möge man zehnmal den Einwand erheben, ich könne es nicht wissen, weil ich nicht, dabei gewesen" sei. Nein, ich war nicht dabei. Aber heute ist seelische Tatsache, daß ich dabei gewesen bin, denn wer eine Geschichte erlebt und wieder durchlebt hat, wie ich diese hier, den macht seine furchtbare Intimität zum Augen- und Ohrenzeugen auch ihrer verborgenen Phasen. (Mann 1947, S. 629) $)^{24}$

In der Forschung werden solche Kommentare Zeitbloms zu seiner Erzählweise zumeist umstandslos als schlagendes Argument für seine Unzuverlässigkeit gewertet; damit wird jedoch erheblich überschätzt, was sich aus ihnen für die Einordnung des Faustus-Erzählers folgern lässt. Für sich genommen nämlich liefern die betreffenden Passagen keine Antwort auf die Frage, ob Zeitblom zuverlässig oder unzuverlässig berichtet; denn sie lassen sich, je nach Gesamtbild, in dem sie betrachtet werden, entweder als Beleg für die Eigentümlichkeit seines Berichts oder aber als Beitrag zu deren Erklärung deuten. ${ }^{25}$

Die Standardeinordnung Zeitbloms verkennt aber nicht allein die Deutungsoffenheit seiner Erzählkommentare; sie geht zudem von einem zu weiten Unzuverlässigkeitsbegriff aus. Ein Erzähler wird nicht dadurch mimetisch unzuverlässig, dass er Ereignisse interpretiert, sondern dadurch, wie er dies tut - genauer gesagt dadurch, dass seine Interpretationen falsch oder aber in markanter Weise zweifelhaft sind. Um Zeitblom überzeugend als mimetisch unzuverlässig einzustufen, reicht es darum nicht aus, zu rekonstruieren, was er selbst offenlegt, nämlich dass er einige der dargestellten Ereignisse nicht erlebt, sondern sich vorgestellt, erschlossen und ausgemalt hat. Eine Argumentation für die Unzuverlässigkeit des Faustus-Erzählers müsste verdeutlichen, dass er die Ereignisse durch seine interpretierende Darstellung signifikant verzerrt.

So aber ist der Erzähler Zeitblom nicht angelegt. In seinen Ausführungen wird weder schlagartig noch schrittweise erkennbar, dass er seine Leser auf eine falsche Fährte zu locken versucht, sich in Widersprüche verstrickt oder Irrtümern aufsitzt. Als Verfasser einer literarisch ausgerichteten Lebensgeschichte legt er einen individuell gefärbten und stellenweise interpretativ ergänzten Bericht vor. Die Konjekturen, die er vornimmt, werden von ihm jedoch nicht nur markiert, sie fallen zudem sehr vorsichtig aus und beziehen sich auf ornamentale Einzelheiten seiner Ausführungen; in zentralen Fragen zur Deutung des Dargestellten hält er sich mit Urteilen auffallend zurück. Musterhaft zeigt sich dies in der Art und Weise, in der er über den Pakt zwischen Leverkühn und dem Teufel berichtet: Das tatsächliche oder eingebildete Gespräch des Tonsetzers mit dem Leibhaftigen übernimmt Zeitblom „Wort für Wort“ (Mann 1947, S. 365) aus den Aufzeichnungen Leverkühns und fügt es als 40-seitiges Zitat in seine eigene

\footnotetext{
${ }^{24} \mathrm{Ganz}$ entsprechend merkt Zeitblom an anderer Stelle an: „Zweifelt jemand, daß ich ,dabei gewesen' bin? Ich denke nicht. Aber ich denke auch, ein genaues Ausbreiten des Vorganges ist für niemanden erforderlich, oder nur wünschbar“ (Mann 1947, S. 641).

${ }^{25}$ Was sich aus den fraglichen Passagen plausibel ableiten lässt, ist nur der negative Befund, dass Zeitblom kein ,täuschend unzuverlässiger` Erzähler ist, vgl. zu diesem Konzept Stühring (2011).
} 
Erzählung ein, ohne eine Einordnung des Geschilderten als natürliches oder übernatürliches Geschehen vorzuschlagen. ${ }^{26}$

Dass sich Zeitblom in seinen Schilderungen zunehmend imaginative Freiheiten gestattet, lässt sich fiktionsintern vor dem Hintergrund der zusammengetragenen Befunde einleuchtender als Ausdruck eines schriftstellerischen Entwicklungsprozesses verstehen denn als Täuschungsversuch, Anmaßung oder Verirrung. Konfrontiert mit der Frage, ob er mit Zeitblom nicht eine inkohärente Erzählerfigur geschaffen habe, hat Mann in einem Brief an Andre Gronicka aus dem Jahr 1948 selbst eine entsprechende Deutung vorgeschlagen:

Sie haben recht, zu sagen, daß Zeitblom aus seiner ursprünglichen Rolle fällt, oder, wenn nicht fällt, ihr doch ein bißchen entgleitet. Und doch ist eigentlich nicht zu sagen, wo er seine Linie verläßt, und wenn er auf ihr zu weit gelangt - könnte man nicht zur Rechtfertigung an eine Art von fieberhafter ,Steigerung ' denken, die ihn im Lauf seines Buchs über sich selbst in Furcht und Zittern hinausführt. (Mann, zitiert nach Wysling und Fischer 1981, S. 202) ${ }^{27}$

Worauf es sich fiktionsextern zurückführen lässt, dass der Doktor Faustus eine solche „Steigerung“ seines Erzählers vorführt, soll im Zusammenhang der nun folgenden Abwägungen zur axiologischen Zuverlässigkeit Zeitbloms betrachtet werden.

Axiologische Zuverlässigkeit? Wie die mimetische Angemessenheit der Leverkühn-Biographie Zeitbloms so wird auch deren axiologische Musterhaftigkeit für den Faustus-Roman vielfach in Zweifel gezogen. Die vom Erzähler in seinem Bericht vertretenen Werthaltungen, so die in der Forschung mittlerweile vorherrschende Einschätzung, kommen nicht mit denen zur Deckung, die das Werk zum Ausdruck bringt. ${ }^{28}$ Dass dies grundsätzlich der Fall ist, lässt sich im vorliegenden Kontext nicht eingehend verdeutlichen. Da Jens Ewen und Ludwig Stockinger $^{29}$ das ,komplizierte Verhältnis zwischen den unterschiedlichen Wertesystemen von Erzähler, Held und Autorinstanz" ${ }^{30}$ im Doktor Faustus in neueren Beiträgen differenziert erläutert haben, kann es hier aber auch genügen, die Unterschiede zwischen der Axiologie Zeitbloms und der des Textes beispielhaft vor Augen zu führen.

\footnotetext{
${ }^{26}$ Wie seit seinen Anfängen in vielen Werken spielt Mann auch im ,Teufelsgespräch'-Kapitel mit Verfahren der literarischen Phantastik (vgl. zum Zusammenhang die Beiträge in Sprecher und Wimmer 2011). Auch wenn die betreffenden Romanpassagen grundsätzlich so angelegt sind, dass sie die ,Ungewissheit“ über den Realitätsstatus des Dargestellten hervorrufen, die Tzvetan Todorov als Kennzeichen des Phantastischen beschrieben hat, so wird eine entsprechende Wirkung doch durch die insgesamt allegorische Konzeption des Doktor Faustus (s. hierzu unten) stark beeinträchtigt (vgl. Kindt 2011, S. 54 f.).

${ }^{27}$ Den Anspielungen in Manns Briefbemerkung, die unter anderem auf seine Princetoner Zauberberg-Lecture und die Philosophie Sören Kierkegaards Bezug nimmt, kann hier nicht nachgegangen werden.

${ }^{28}$ Vgl. etwa Petersen (2008), Baier (2011) oder Ewen (2014).

${ }^{29} \mathrm{Vgl}$. Ewen (2014) und Stockinger (2017).

${ }^{30}$ Stockinger (2017, S. 223).
} 
Markant treten diese Differenzen zutage, wenn man einige ZeitblomKommentare zu den historischen Entwicklungen der 1930er und 1940er Jahre vor dem Hintergrund des gesamten Romans betrachtet. Was Zeitblom in seinen Aufzeichnungen zum Nationalsozialismus, zu Deutschland und zu dessen Rolle im Zweiten Weltkrieg notiert, lässt zwar zumeist eine eher distanzierte Haltung erkennen, es fällt vielfach aber weniger kritisch aus, als es der Doktor Faustus im Ganzen nahe legt. Dies gilt etwa für Zeitbloms Bemerkung, dass er ,in der Judenfrage und ihrer Behandlung unserem Führer und seinen Paladinen niemals voll habe zustimmen können“ (Mann 1947, S. 17), oder für seine Einschätzung, dass der Krieg ,den Intellektuellentraum eines europäischen Deutschland durch die allerdings etwas beängstigende, etwas brüchige und, wie es scheint, der Welt unerträgliche Wirklichkeit eines deutschen Europa ersetzt" (Mann 1947, S. 251) habe. Wie auch immer man die Wertordnung des Faustus-Romans im Einzelnen bestimmen mag, entsprechende Kommentare signalisieren, dass das Wertesystem der Erzählerfigur nicht mit dem des Werks zusammenfällt und dass es darum gerechtfertigt ist, sie als Hinweise auf Zeitbloms axiologische Unzuverlässigkeit zu deuten.

Mit Blick auf die Erzählanlage des Romans ist ein solches Urteil, so angemessen es grundsätzlich sein mag, freilich nur bedingt erhellend. Das liegt daran, dass ,axiologische Unzuverlässigkeit' ein im Anschluss an Booth recht weit gefasstes Konzept ist, das eine Reihe mehr oder weniger unterschiedliche Instantiierungen haben kann. ${ }^{31}$ Konkret gesagt: Zeitbloms Klassifikation als , axiologisch unzuverlässig ' besagt nur, dass sich im Doktor Faustus Erzähler- und Werknormen voneinander abheben, sie lässt aber offen, wie dies genau umgesetzt ist. Auf eben dieses ,Wie' kommt es im Fall von Manns Roman jedoch an; denn bei Zeitblom handelt es sich in mindestens zwei Hinsichten um ein untypisches Beispiel für einen axiologisch unzuverlässigen Erzähler.

Der Unzuverlässigkeitsbefund ist zunächst um die Beobachtung zu ergänzen, dass im Verlauf der Aufzeichnungen neben der Präsentationsweise auch das zum Ausdruck gebrachte Wertesystem eine Entwicklung durchläuft. ${ }^{32}$ Musterhaft zeigt sich dies wiederum in Zeitbloms Anmerkungen zu den geschichtlichen Entwicklungen seit der Zeit der Weimarer Republik. In den Ausführungen kommt es zunehmend zu Stellungnahmen, die sich besser in die Axiologie des Werks einfügen als die oben angeführten Kommentare. Nicht wenige der Überzeugungen, die Zeitblom in späteren Kapiteln seiner Erzählung vertritt, hat Mann dabei im Rückgriff auf Positionen und Formulierungen aus eigenen Texten der 1940er Jahre gestaltet. ${ }^{33}$ „Heute sieht man wohl“", so lässt er seinen Erzähler etwa zum

\footnotetext{
${ }^{31}$ Vgl. dazu die Unzuverlässigkeitsbestimmung von Booth in Teil 1 und den Klärungsvorschlag in Köppe und Kindt (2014, S. 250-253).

${ }^{32} \mathrm{Vgl}$. hierzu die genaue Analysen in Kaiser (2001), Kap. 3.3.

${ }^{33}$ Vgl. Stockinger (2017, S. 222).
} 
Verhalten gegenüber den Nationalsozialisten anmerken, „daß es der Fehler unserer Zivilisation war, diese Schonung und diesen Respekt allzu hochherzig geübt zu haben, wo sie es doch auf der Gegenseite mit barer Frechheit und der entschlossensten Intoleranz zu tun hatte“ (Mann 1947, S. 387). Doktor Faustus präsentiert einen Erzähler, so ist festzuhalten, dessen Werthaltungen während seines Berichts tendenziell zuverlässiger werden. ${ }^{34}$

Damit ist die axiologische Unzuverlässigkeit Zeitbloms genauer bestimmt, die Analyse muss aber noch um einen präzisierenden Hinweis erweitert werden, der die Stellung seiner Wertauffassungen im Kontext der Wertordnung des Romans betrifft. Wenn die Normen eines Erzählers - mit Booth gesprochen nicht ,in accordance“ mit den Normen des Werks sind, dann heißt das oft, dass sich zwischen den betreffenden Wertordnungen ein Spannungsverhältnis ausmachen lässt; sie stehen, kurz gesagt, im Widerspruch zueinander. Die Rede von mangelnder ,accordance“ kann aber auch bedeuten, dass die betrachteten Normgefüge nicht miteinander in Übereinstimmung sind. In diesem Fall muss zwischen den Axiologien von Erzähler und Werk kein grundlegender Konflikt bestehen; ihre fehlende Übereinstimmung kann auch darin bestehen, dass sie einander nur punktuell widersprechen oder durch eine divergierende bzw. unterschiedlich hierarchisierte Auswahl von Werten charakterisiert sind. ${ }^{35}$ Dies scheint die Variante axiologischer Unzuverlässigkeit zu sein, die in den späteren Kapiteln des Doktor Faustus realisiert ist. ${ }^{36}$

Wie Ewen gezeigt hat, lässt sich Manns Roman als ein Beitrag zum literarischen Modernediskurs verstehen, der anhand der Kunst- und Kulturverständnisse seiner Protagonisten die Unzulänglichkeit verschiedener grundlegender Haltungen zur Modernisierung und auf diese Weise die Bedeutung eines gesellschaftlichen Pluralismus zur Anschauung bringt. ${ }^{37}$ Bei Leverkühn und Zeitblom handelt es sich um Figuren, die zugleich eine ,Symbolfunktion ${ }^{\text {‘3 }}$ besitzen; sie repräsentieren unterschiedliche, aber gleichermaßen einseitige Einstellungen zum fundamentalen Wandel der Gesellschaft in der Neuzeit. Leverkühn verkörpert, stark vereinfacht gesagt, die Sehnsucht nach der Auflösung des Individuums in einem größeren Ganzen, Zeitblom die Hoffnung auf Bewahrung

\footnotetext{
${ }^{34}$ Es kann hier nicht diskutiert werden, ob die alltagssprachlich unproblematische Rede von der „abnehmenden“ bzw. „zunehmenden Zuverlässigkeit“ eines Erzählers auch literaturwissenschaftlich und also fachsprachlich sinnvoll ist. Im Rahmen dieses Beitrags wird „unzuverlässig erzählt" - wie in der Literaturwissenschaft zumeist - nicht als komparatives, sondern als klassifikatorisches Prädikat verwendet, das Werken im Ganzen zugeschrieben wird.

${ }^{35}$ Bei den Erzählernormen kann es sich also z. B. auch um eine Teilmenge der Werknormen handeln.

${ }^{36}$ Die beiden unterschiedenen Varianten sind nur zwei idealtypische der vielen möglichen Formen, in denen Erzähler- und Werknormen voneinander abweichen können.

${ }^{37} \mathrm{Vgl}$. Ewen (2014).

${ }^{38}$ Vgl. dazu Köppe und Kindt (2014, S. 146-148).
} 
des Humanen durch Kultur. ${ }^{39}$ Der Faustus-Roman erkundet über Leverkühn und Zeitblom aber nicht allein diese Haltungen, sondern auch deren Zusammenhang; er führt vor, dass die Grundorientierungen der Hauptfiguren aufeinander bezogen sind, aber unvermittelt bleiben - und nicht zuletzt deshalb zur „Katastrophendynamik“ (Mann 1947, S. 438) des 20. Jahrhunderts beitragen. In Erläuterungen zu der Schwierigkeit, die allegorisch angelegten Protagonisten des Romans zu charakterisieren, hat Mann deren Verbindung mit der seither vielzitierten Formel vom „Geheimnis ihrer Identität“" umschrieben:

Ein Verbot war hier einzuhalten - oder doch dem Gebot größter Zurückhaltung zu gehorchen bei einer äußerster Verlebendigung, die sofort den seelischen Fall und seine Symbolwürde, seine Repräsentativität mit Herabsetzung, Banalisierung bedrohte. Es war nicht anders: Romanfiguren im pittoresken Sinn durften nur die dem Zentrum ferneren Erscheinungen des Buches [...] sein - nicht seine beiden Protagonisten, die viel zu verbergen haben, nämlich das Geheimnis ihrer Identität. (Mann 1949, S. 473 f.; Hervorhebungen im Original)

Es kann hier offen bleiben, wie das Verhältnis zwischen den beiden Hauptfiguren des Romans und den durch sie symbolisch zum Ausdruck gebrachten Haltungen näher zu rekonstruieren ist. Festzuhalten ist aber, dass das Wertesystem des Doktor Faustus weder einfach ex negativo aus den axiologischen Orientierungen seiner Protagonisten $\mathrm{zu}$ erschließen ist noch in einer von ihnen ein figurales Sprachrohr findet. Offensichtlich ist dies im Fall der Werthaltungen Leverkühns, es gilt aber auch für diejenigen Zeitbloms. In seiner Erzählung mag er zunehmend Einschätzungen und Einstellungen zum Ausdruck bringen, die im Zusammenhang des Werkganzen nicht als unangemessen erkennbar werden, sondern nachvollziehbar oder sogar bedenkenswert erscheinen. Er gelangt jedoch nicht zu einer Haltung der Selbstkritik, Pluralismusbejahung und Moderneoffenheit, wie sie der Doktor Faustus als Konsequenz aus den Verbrechen und dem Leid des 20. Jahrhunderts nahe legt. ${ }^{40}$ Inwiefern er beigetragen hat zu den „Schrecknissen der Zeit“ (Mann 1947, S. 729), bleibt Zeitblom auch nach dem Abschluss seines Berichts verborgen.

Im Zuge seiner Schilderungen, so lässt sich zusammenfassen, wächst nicht allein Zeitbloms Fabulierlust, es kommt zugleich zur Schärfung seines Urteilsvermögens. Beide Züge seiner Entwicklung wirken sich allerdings, anders als oft angenommen wird, auf seine Zuverlässigkeit als Erzähler nicht entscheidend aus. Dass er zunehmend romanhaft erzählt, disqualifiziert ihn nicht als Biographen; dass er nach und nach reflektierter urteilt, lässt ihn nicht zum Sprachrohr der Werknormen avancieren. Ungeachtet seiner Wandlung bleibt er ein mimetisch zuverlässiger, aber axiologisch unzuverlässiger Erzähler. Die betrachtete

\footnotetext{
${ }^{39}$ Hierin geht die Symbolfunktion der Protagonisten bekanntlich nicht auf; sie lassen sich zudem als allegorische Thematisierungen von Charakteristika interpretieren, die Thomas Mann sich selbst und den Deutschen zugeschrieben hat, vgl. zu dieser Dimension der Hauptfiguren, der hier nicht nachgegangen werden kann, Reed (1993) und Vaget (2015).
}

${ }^{40} \mathrm{Vgl}$. dazu insbesondere Ewen (2014, S. 282 f.). 
Entwicklung, die Mann selbst als „Steigerung“"41 beschrieben hat, ist fiktionsintern überzeugend auf die Erfahrungen zurückzuführen, die Zeitblom durch sein Erzählvorhaben und dessen sich wandelnde Umstände im nationalsozialistischen Deutschland der letzten Weltkriegsjahre gewinnt; fiktionsextern erklärt sich die Metamorphose des Erzählers im Doktor Faustus wesentlich daraus, dass Mann das ,Medium“ Zeitblom, das er ursprünglich zur ,Durchheiterung` des Werks eingeführt hatte, während der Ausarbeitung des Romans sowohl literarisch als auch konzeptionell zunehmend als Einengung wahrnahm. ${ }^{42}$ Durch die Entwicklung seines Erzählers suchte Mann - mehr oder weniger bewusst - sicher zu stellen, dass er sich auch in der Rolle Zeitbloms schriftstellerisch entfalten und erzählerische Virtuosität zur Schau stellen konnte. Zugleich erleichterte ihm die sukzessive Wandlung der Erzählerfigur, dem Werk eine komplexere Wirkungskonzeption zu geben, die neben die ironische Entlarvung von Positionen und Perspektiven deren ambivalentes Nebeneinander treten ließ.

Die vorstehenden Überlegungen haben $\mathrm{zu}$ verdeutlichen versucht, dass es unangemessen und irreführend ist, Serenus Zeitblom aus Thomas Manns Doktor Faustus als ,unzuverlässigen Erzähler" zu beschreiben. Textanalytisch mag es gerechtfertigt sein, ihn in einer Hinsicht und eigenwilligen Ausprägung als unzuverlässig zu klassifizieren; in literaturgeschichtlicher und erzählpoetischer Perspektive aber ist für Manns Roman gerade eine besondere Form von erzählerischer Zuverlässigkeit kennzeichnend - eine, die nicht einfach vorausgesetzt werden kann oder offensichtlich erscheint und darum im Zuge der Lektüre rekonstruiert werden muss. Durch die detaillierte Charakterisierung dieser Zuverlässigkeitsspielart im Doktor Faustus sollte daran erinnert werden, dass sich ein gehaltvolles Verständnis von erzählerischer Unzuverlässigkeit nur dann gewinnen lässt, wenn sie im Zusammenhang mit und in Abgrenzung von anderen Verfahren und Kunstgriffen literarischer Narration betrachtet wird. Wie die Analyse von Zeitblom in diesem Sinne vor Augen geführt hat, muss Rollenprosa, also das Erzählen mit figürlich ausgestalteten Vermittlungsinstanzen ebenso wenig auf Unzuverlässigkeit hinauslaufen wie der Einsatz ironisierter, interpretierender oder imaginierender Erzählerfiguren.

\section{Literatur}

Andersch, Alfred: Deutsche Literatur in der Entscheidung. Ein Beitrag zur Analyse der literarischen Situation. Karlsruhe 1948.

Baier, Christian: ,...ich weiß es, und möge man zehnmal den Einwand erheben, ich könne es nicht wissen...". Analyse einer Szene in Thomas Manns Roman Doktor Faustus. In: Wirkendes Wort 2008, S. 113-126.

\footnotetext{
${ }^{41}$ Siehe oben.

${ }^{42} \mathrm{Zu}$ ähnlichen Erklärungsansätzen vgl. etwa Schulze-Berge (2006, S. 169-194) oder Göbel (2015, S. 121-127).
} 
Baier, Christian: Zwischen höllischem Feuer und doppeltem Segen. Geniekonzepte in Thomas Manns Romanen „Lotte in Weimar", „Joseph und seine Brüder" und „Doktor Faustus“. Göttingen 2011.

Booth, Wayne C.: The Rhetoric of Fiction [1961]. 2. Aufl. Chicago, London 1983.

Ewen, Jens: Deutungsangebote durch Sympathiepunkte. Zur Strategie narrativer Unzuverlässigkeit in Thomas Manns Roman Doktor Faustus. In: Claudia Hillebrandt, Elisabeth Kampmann (Hg.): Sympathie und Literatur. Zur Relevanz des Sympathiekonzeptes für die Literaturwissenschaft. Berlin 2014, S. 270-283

Fludernik, Monika: An Introduction to Narratology. London/New York 2009.

Göbel, Eckart: Esmaralda. Deutsch-französische Verhältnisse in Thomas Manns „Doktor Faustus “. Göttingen 2015.

Von Kahler, Erich: The Secularization of the Devil: Thomas Mann’s „Doktor Faustus“ [1948]. In: E. v. K.: The Orbit of Thomas Mann. Princeton, NJ 1969, S. 20-48.

Kaiser, Gerhard: „....und sogar eine alberne Ordnung ist immer noch besser als gar keine." Erzählstrategien in Thomas Manns Doktor Faustus. Stuttgart, Weimar 2001.

Kindt, Tom: Turlupin. Oder: Und wo bleibt das Ethische, Herr Perutz? In: Tom Kindt, Jan Christoph Meister (Hg.): Leo Perutz' Romane. Tübingen 2007, S. 69-80.

Kindt, Tom:Unzuverlässiges Erzählen und literarische Moderne. Eine Untersuchung der Romane von Ernst Weiß. Tübingen 2008.

Kindt, Tom: „Das Unmögliche, das dennoch geschieht“. Zum Begriff der literarischen Phantastik am Beispiel von Werken Thomas Manns. In: Thomas Mann-Jahrbuch 24 (2011), S. 43-56.

Kindt, Tom: Narratologie. In: Andreas Blödorn, Friedhelm Marx (Hg.): Thomas Mann-Handbuch. Leben - Werk - Wirkung. Stuttgart 2015, S. 252-255.

Koch, Jonas: Erklären und Verstehen fiktionaler Filme. Semantische und ontologische Aspekte. Paderborn 2015.

Köppe, Tilmann/Kindt, Tom: Unreliable Narration with a Narrator and Without. In: JLT Journal of Literary Theory 5:1 (2011), S. 37-50

Köppe, Tilmann/Kindt, Tom: Erzähltheorie. Eine Einführung. Stuttgart 2014.

Löwe, Matthias: Unzuverlässigkeit bei heterodiegetischen Erzählern. Konturierung eines Konzepts an Beispielen von Thomas Mann und Goethe. In: JLT - Journal of Literary Theory 12:1 (2018), S. 77-92.

Mann, Thomas: Doktor Faustus. Das Leben des deutschen Tonsetzers Adrian Leverkühn, erzählt von einem Freunde [1947]. Hg. von Ruprecht Wimmer unter Mitarbeit von Stephan Stachorski. Frankfurt a. M. 2007 (Thomas Mann - Große kommentierte Frankfurter Ausgabe, Bd. 10.1).

Mann, Thomas:Die Entstehung des Doktor Faustus [1949]. In: T. M.: Essays VI: 1945-1950. Hg. von Herbert Lehnert. Frankfurt a. M. 2009 (Thomas Mann - Große kommentierte Frankfurter Ausgabe, Bd. 19.1), S. 409-581.

Martinez, Matias/Scheffel, Michael: Einführung in die Erzähltheorie. Beck: München 1999.

Mayer, Hans: Die umerzogene Literatur. Deutsche Schriftsteller und Bücher 1945-1967. Berlin 1988.

Neumann, Uwe: Dem Zufall aus dem Wege gehen. Weitere Funde zur Zahlenmystik bei Uwe Johnson. In: Johnson-Handbuch 23 (2016), S. 153-182.

Petersen, Jürgen H.: „Faustus“ lesen. Eine Streitschrift zu Thomas Manns spätem Roman. Würzburg 2007.

Petersen, Jürgen H.: „Der unzuverlässige Narrator. Figuren-Erzählen in Thomas Manns Doktor Faustus. In: Revista di Filología Alemana 16 (2008), S. 167-185.

Reed, Terence James: Doktor Faustus. In: Volkmar Hansen (Hg.): Thomas Mann. Romans und Erzählungen. Stuttgart 1993, S. 294-324.

Riggan, William: Picaros, Madmen, Naives, and Clowns: The Unreliable First-Person Narrator. Norman 1981.

Scheuer, Helmut: Biographie. Literarische Darstellung eines Lebenslaufs. In: Klaus Weimar u.a. (Hg.): Reallexikon der deutschen Literaturwissenschaft. Bd. 1. Berlin/New York 1997, S. 233-236. 
Schmidt-Schütz, Eva: „Doktor Faustus” zwischen Tradition und Moderne: eine quellenkritische und rezeptionsgeschichtliche Untersuchung zu Thomas Manns literarischem Selbstbild. Frankfurt a. M. 2003.

Schulze-Berge, Sibylle: Heiterkeit im Exil. Ein ästhetisches Prinzip bei Thomas Mann: zur Poetik des Heiteren im mittleren und späten Werk Thomas Manns. Würzburg 2006.

Sprecher, Thomas/Wimmer, Ruprecht (Hg.): Thomas Mann Jahrbuch 24. Frankfurt a. M. 2011.

Stockinger, Ludwig: Serenus Zeitblom und Monsignore Hinterpförtner. Darstellung und Deutung des ,Christlichen Humanismus' des Zwischenkriegszeit in Thomas Manns Doktor Faustus. In: Matthias Löwe, Gregor Streim (Hg.): Humanismus in der Krise. Debatten und Diskurse zwischen Weimarer Republik und geteiltem Deutschland. Berlin/Boston 2017, S. 215-234.

Vaget, Hans Rudolf: Thomas Mann, der Amerikaner. Leben und Werk im amerikanischen Exil 1938-1952. Frankfurt a. M. 2012.

Vaget, Hans Rudolf: Doktor Faustus. In: Andreas Blödorn, Friedhelm Marx (Hg.): Thomas Mann-Handbuch. Leben - Werk - Wirkung. Stuttgart 2015, S. 66-75.

Viswathan, Jacqueline: Point of View and Unreliability in Brontë's Wuthering Heights, Conrad's Under Western Eyes and Mann's Doktor Faustus. In: Orbis Litterarum 29:1 (1974), S. 42-50.

Wimmer, Ruprecht/Stachorski, Stephan: Doktor Faustus. Das Leben des deutschen Tonsetzers Adrian Leverkühn, erzählt von einem Freunde. Kommentarband. Frankfurt a.M. 2007 (Thomas Mann - Große kommentierte Frankfurter Ausgabe, Bd. 10.2).

Wysling, Hans/Fischer, Marianne (Hg.): Dichter über ihre Dichtungen: Thomas Mann. Bd. 3: 1944-1955. Zürich, München, Frankfurt a. M. 1981.

Open Access Dieses Kapitel wird unter der Creative Commons Namensnennung 4.0 International Lizenz (http://creativecommons.org/licenses/by/4.0/deed.de) veröffentlicht, welche die Nutzung, Vervielfältigung, Bearbeitung, Verbreitung und Wiedergabe in jeglichem Medium und Format erlaubt, sofern Sie den/die ursprünglichen Autor(en) und die Quelle ordnungsgemäß nennen, einen Link zur Creative Commons Lizenz beifügen und angeben, ob Änderungen vorgenommen wurden.

Die in diesem Kapitel enthaltenen Bilder und sonstiges Drittmaterial unterliegen ebenfalls der genannten Creative Commons Lizenz, sofern sich aus der Abbildungslegende nichts anderes ergibt. Sofern das betreffende Material nicht unter der genannten Creative Commons Lizenz steht und die betreffende Handlung nicht nach gesetzlichen Vorschriften erlaubt ist, ist für die oben aufgeführten Weiterverwendungen des Materials die Einwilligung des jeweiligen Rechteinhabers einzuholen. 